\title{
Level of implementation of best practice policies for creating healthy food environments: assessment by state and non-state actors in Thailand
}

\author{
Sirinya Phulkerd ${ }^{1,2, *}$, Stefanie Vandevijvere ${ }^{3}$, Mark Lawrence ${ }^{4}$, Viroj Tangcharoensathien ${ }^{2}$ \\ and Gary Sacks ${ }^{5}$ \\ ${ }^{1}$ School of Exercise and Nutrition Sciences, Deakin University, 221 Burwood Highway, Burwood, VIC 3125, \\ Australia: ${ }^{2}$ International Health Policy Program, Ministry of Public Health, Nonthaburi, Thailand: ${ }^{3}$ Department of \\ Epidemiology and Biostatistics, School of Population Health, Faculty of Medical and Health Sciences, University of \\ Auckland, Auckland, New Zealand: ${ }^{4}$ Institute for Physical Activity and Nutrition, Deakin University, Burwood, \\ Victoria, Australia: ${ }^{5}$ WHO Collaborating Centre for Obesity Prevention, Deakin University, Burwood, Victoria, \\ Australia
}

Submitted 29 April 2016: Final revision received 26 July 2016: Accepted 1 August 2016: First published online 13 September 2016

\begin{abstract}
Objective: To determine and compare the level of implementation of policies for healthy food environments in Thailand with reference to international best practice by state and non-state actors.

Design: Data on the current level of implementation of food environment policies were assessed independently using the adapted Healthy Food Environment Policy Index (Food-EPI) by two groups of actors. Concrete actions were proposed for Thai Government. A joint meeting between both groups was subsequently held to reach consensus on priority actions.

Setting: Thailand.

Subjects: Thirty state actors and twenty-seven non-state actors.

Results: Level of policy implementation varied across different domains and actor groups. State actors rated implementation levels higher than non-state actors. Both state and non-state actors rated level of implementation of monitoring of BMI highest. Level of implementation of policies promoting in-store availability of healthy foods and policies increasing tax on unhealthy foods were rated lowest by state and non-state actors, respectively. Both groups reached consensus on eleven priority actions for implementation, focusing on food provision in public-sector settings, food composition, food promotion, leadership, monitoring and intelligence, and food trade.

Conclusions: Although the implementation gaps identified and priority actions proposed varied between state and non-state actors, both groups achieved consensus on a comprehensive food policy package to be implemented by the Thai Government to improve the healthiness of food environments. This consensus is a platform for continued policy dialogue towards cross-sectoral policy coherence and effective actions to address the growing burden of non-communicable diseases and obesity in Thailand.
\end{abstract}

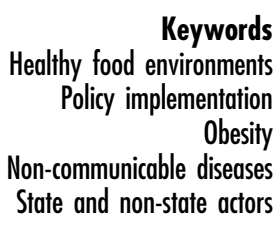

The WHO recommendations for the control and prevention of non-communicable diseases (NCD) include establishing and strengthening country-level surveillance and monitoring as a top priority ${ }^{(1)}$. In recent years there have been increasing attempts to monitor and evaluate government policy responses to address obesity and NCD.

Often, such monitoring has been performed by WHO or global non-governmental organizations (NGO) such as the World Cancer Research Fund and World Obesity, rather than country governments. The WHO's monitoring system specifically focuses on health outcomes, NCD risk factors and national system responses, but includes aspects of food environments, which are significant contributors to obesity and diet-related NCD, in only a limited way ${ }^{(2-4)}$. The WHO monitoring system includes two indicators on food environment policies: one on reduction of food marketing to children and one on limiting saturated and trans-fats in the food supply ${ }^{(5)}$. 
Involving diverse stakeholders, including state and non-state actors, in the monitoring and evaluation of policy implementation is important to increase awareness, accountability and commitment on policy outcomes and impacts $^{(6)}$ as well as to gain support for the implementation of proposed priorities. A self-assessment by state actors can help to set baselines, identify gaps, plan and prioritize actions, and increase commitment to addressing the issue under assessment; for example, the assessment through WHO's monitoring system on $\mathrm{NCD}^{(5)}$ and alochol $^{(7)}$. Non-state actors' involvement in the assessment can help to promote government policies and actions by raising their awareness, encouraging their acceptance and increasing their knowledge on the policies and actions ${ }^{(6)}$. Creating a platform for interactions between state and non-state actors is useful to identify areas of commonality for reconciling differences associated with a particular issue. To be effective, assessing the implementation of recommended policies by the government, therefore, should be undertaken as a multi-stakeholder process.

The objectives of the present study were to: (i) engage government officials (simply called 'state actors') and nongovernmental experts (simply called 'non-state actors') to do an assessment of the level of implementation of recommended food environment policies against international best practice; (ii) propose and prioritize actions to improve the healthiness of Thai food environments; (iii) compare the findings on the implementation gaps and actions proposed and prioritized by state and non-state actors; and (iv) seek consensus between state and non-state actors on the priority actions to be implemented by the Thai Government.

\section{Methods}

The Healthy Food Environment Policy Index (Food-EPI) tool and process from the International Network for Food and Obesity/NCDs Research, Monitoring and Action Support (INFORMAS) ${ }^{(8)}$ were adapted and made applicable to the Thai context. The Food-EPI includes two components (food policies and infrastructure support), thirteen domains and forty-two good practice indicators (nineteen food policy indicators and twenty-three infrastructure support indicators; see online supplementary material, Table S1). Two steps were added to the original Food-EPI process, namely (i) checking appropriateness of domains and good practice indicators of the Food-EPI for the Thai context and (ii) translating the indicators of the Food-EPI into the local language. The adapted Food-EPI process included ten key steps (Fig. 1). The international best practices used as benchmarks in the present study were compiled by INFORMAS through deriving information from the World Cancer Research Fund NOURISHING database $^{(9)}$ complemented with other best practice examples received from international experts on food and nutrition and obesity policy.
First, all food environment domains and indicators of the version of the Food-EPI used previously were verified with two Thai experts: one from the Ministry of Public Health and one from the Health Promotion Policy Research Center of the International Health Policy Program Foundation, in order to ensure the indicators were appropriate for Thailand. All of the Food-EPI indicators were found to be applicable to the Thai context and no new ones were added. Then, the indicators were translated from English to Thai by S.P. and translated back to English by an independent person. The backtranslation was used to check discrepancy between the original and translated versions.

\section{Evidence compilation and validation}

Evidence for implementation was gathered for all fortytwo indicators of the Food-EPI. Government documents and budget information related to food environment policy were collected from data sources such as official information requests, governmental websites and libraries, NGO publications and websites, major Thai newspapers, and via contact with government officials. Data were collected in relation to current government policy, defined for the purposes of the present study as policies in place from October 2014 (time when the current Cabinet was formed) to October 2015 (the date at which evidence collection for the project finished). After gathering all evidence, the completeness and accuracy of the evidence gathered were verified by government officials. Meetings were held with all nine government officials (one each from the Office of Agricultural Economics, Ministry of Agriculture and Cooperative; the Department of Health, the Bureau of Nutrition, the Food and Drug Administration Office and the Bureau of Policy and Strategy, Ministry of Public Health; the Bureau of Student Activity Development, Ministry of Education; and the Excise Department, Ministry of Finance; and two from the National Health Commission Office of Thailand), supplemented with personal email communications with individual officials on some specific issues.

After compiling and validating the evidence, twelve indicators out of the forty-two Food-EPI indicators were excluded from the rating process, since the level of implementation of those was considered by the government officials to be nil. Ratings were performed for thirty indicators only, including eleven food policy indicators and nineteen infrastructure support indicators.

\section{Pilot test}

The rating process using the translated Food-EPI and the validated evidence was pilot tested with ten experts from NGO and academic institutes. Two observations per participant (four weeks apart) were conducted to detect the consistency in the data gathered within and between raters. 


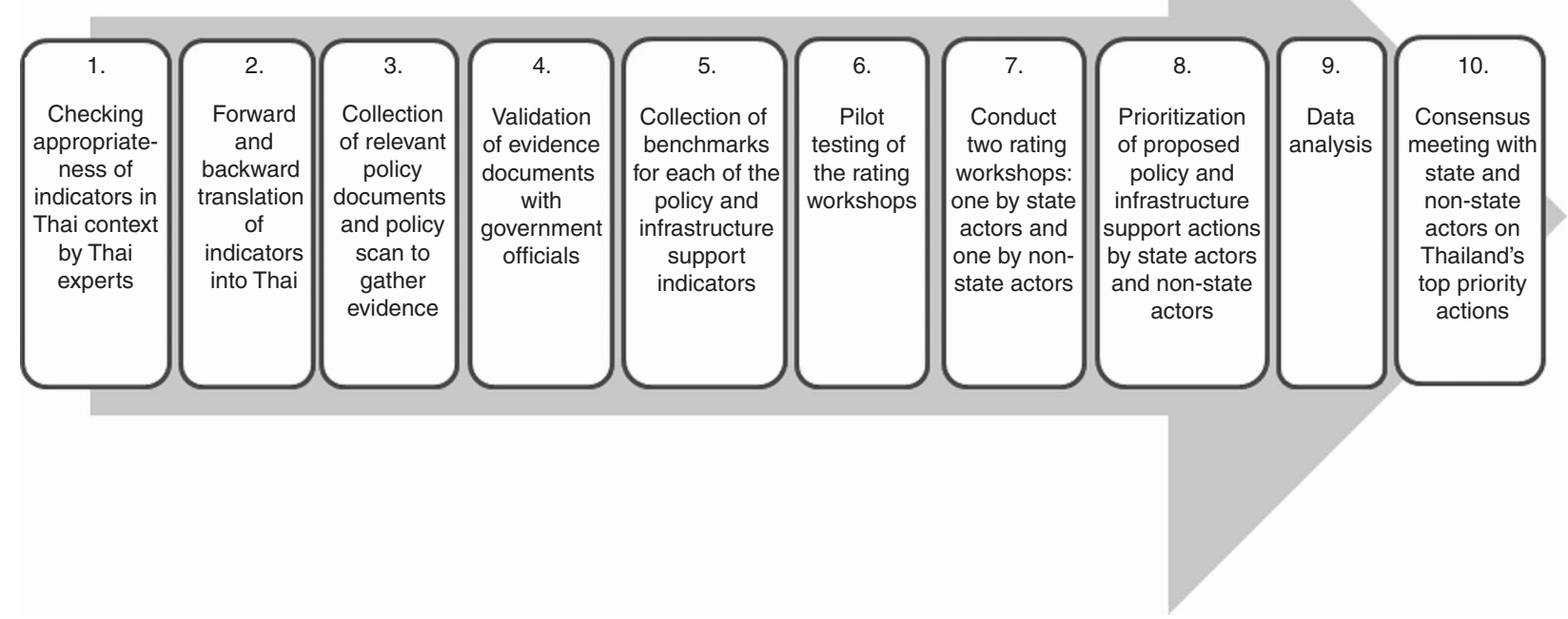

Fig. 1 The ten-step process for assessing the implementation of food environment policies and infrastructure support for policy implementation in Thailand (adapted from Swinburn et al. ${ }^{(8)}$ )

\section{Rating workshop}

A one-day rating workshop was conducted separately with state and non-state groups. The self-assessment of the policy implementation by the state actors is a novel addition to the traditional Food-EPI study which is usually conducted with a non-governmental expert panel only. An initial list of state and non-state actors to invite to the workshop was compiled based on information from secondary data sources using the same approach as that used for compiling the government evidence. This list was then refined to ensure that a variety of stakeholders was represented. The final list of invitees included state actors from across government sectors (regulators and implementers from central and local administrations) and public health experts from Thai universities, research institutes or NGO on food and nutrition, public health, health policy and family network (senior advisors, university professors, directors, researchers or consumer advocates). During the workshop the evidence and benchmarks were presented to these participants for each indicator. The participants rated the current level of government policy implementation in Thailand against international best practices using Likert scales from 1 to 5 ( $1=<20 \%$ implemented, $2=20-40 \%$ implemented, $3=40-60 \%$ implemented, $4=60-80 \%$ implemented, $5=80-100 \%$ implemented compared with best practice).

\section{Prioritization}

Based on the implementation gaps identified, the actors at the workshop then proposed concrete actions to be implemented by the Thai Government. All proposed actions were listed and considered for prioritization. After the workshop, the participants were asked to score importance and achievability for each of the proposed actions, and then the research team analysed the average scores of each action, as per the Food-EPI protocol ${ }^{(8)}$.

An initial score for each action was set at 5 for both importance and achievability, but the participants could adjust these scores for each action. However, for each participant, the sum scores of all actions for both importance and achievability had to equal the multiplication of 5 and the number of actions. In this case, as there were sixty policy actions proposed, each action had an initial score of 5 points but the experts could give any score to each action, so long as the total score for all actions was $5 \times 60=300$.

\section{Stakebolder meeting}

After completion of the Food-EPI assessment, the rating and prioritization results from state and non-state actors were reported to all actors through a stakeholder meeting between state and non-state actors. The meeting aimed to seek consensus on the priority actions to be implemented by the Thai Government to improve the healthiness of food environments through taking into account the views of all actors concerned and to reconcile any conflicting arguments and findings between both groups.

The stakeholder meeting was hosted and facilitated by the research team (V.T. and S.P.) including the following steps: (i) the participants were invited to ask questions and request clarification about the project before the discussion started; (ii) the research team presented four-quadrant scatter plots of the prioritized actions for both state and non-state actors - each quadrant represented a group of 
actions with high/low degree of importance and achievability; (iii) the participants reviewed the actions in each quadrant, beginning with the 'relatively higher importance and achievability' group of actions, and then had a discussion with the view of reaching consensus on the priority actions; (iv) the participants discussed if any other actions (not previously discussed) should be included as a priority; and (v) the participants approved a set of consensus priority actions that the Thai Government should take further in responding to obesity and diet-related NCD.

\section{Data analysis}

For each group, descriptive statistics (average and percentage) were computed to examine (i) ratings on the level of implementation of each policy and infrastructure support indicator and (ii) prioritization scores for importance and achievability of policy actions and infrastructure support actions. The rating scores were categorized into four implementation levels: high (>75\% implemented), medium (51-75\% implemented), low (26-50\% implemented) and very little, if any ( $<25 \%$ implemented) against international best practice. The twelve good practice indicators, for which no ratings were performed, were included under 'very little, if any implementation'. Assessment of inter-rater reliability using the Gwet AC2 statistic was performed to measure the degree to which different actors in each group agreed in their assessment decisions for each of the indicators when using the Food-EPI. Inter-rater reliabilities were $0.735(95 \%$ CI $0.66,0.81$; percentage agreement $=91 \%)$ and $0.794(95 \%$ CI $0.73,0.85$; percentage agreement $=$ $92 \%)$ in the first and second pilot study, respectively. Intrarater reliability was 0.92 . The inter-rater reliability among state actors was also assessed. The overall score was 0.73 (95\% CI 0.69, 0.77; percentage agreement $=91 \%$ ).

Differences between the ratings of state actors and the ratings of non-state actors were then compared. First, the distribution of the data was tested using the Shapiro-Wilk test. The data were found to be non-normally distributed and therefore the Wilcoxon rank-sum test was conducted to assess whether there was a significant difference between groups.

For each group (state and non-state actors), the prioritizing scores were summed for each criterion (importance and achievability) for each proposed action, and then an average score was calculated. The average importance and achievability score of each action was posted on a four-quadrant scatter graph and the actions were divided into four groups: (i) 'relatively higher importance and relatively higher achievability' group; (ii) 'relatively higher importance and relatively lower achievability' group; (iii) 'relatively lower importance and relatively higher achievability' group; and (iv) 'relatively lower importance and relatively lower achievability' group. The points dividing the graph into the quadrants were calculated by summing the average score of all actions in each criterion and then dividing this sum by the total number of actions.

\section{Results}

Forty-eight state actors and forty-six non-state actors were invited to the workshop. Thirty state actors and twenty-seven non-state actors attended the full day workshop. The state actors were from eight Ministries (Public Health, Education, Finance, Commerce, Industry, Agriculture and Cooperation, Interior, and Information and Communication Technology) and three other public organizations (National Economic and Social Development Board, Bangkok Metropolitan Office and Border Patrol Office of Royal Thai Police). Out of thirty state actors, eighteen participants held the position of Director, Deputy Director or senior professional level, and twelve were in the position of professional or practitioner level, or equivalent. Out of twenty-seven non-state actors, seven participants were researchers at universities, eleven were from NGO, and nine indicated that they worked as a researcher at a university while also holding at least one position in an NGO.

\section{Rating the level of government policy implementation in Thailand against international best practice}

Figure 2 illustrates the comparison of overall percentage for each indicator categorized into four implementation levels by state and non-state actors. Among the state actors, the level of implementation of infrastructure support systems for policy implementation was rated higher than the implementation of food environment policies. The level of implementation compared with best practice was assessed as 'high' for five indicators and 'medium' for twenty-five indicators (Fig. 2). Among the food policy domains, the extent of implementation of ingredient lists and nutrient declarations was rated highest (LABEL1; 76.7\%) and the extent of implementation of in-store availability of healthy foods lowest (RETAIL2; 54.0\%). Among the infrastructure support domains the state actors assessed their performance on monitoring nutrition status and population intake and monitoring overweight and obesity as high (MONIT2, MONIT3; $77.3 \%$ ) and cross-sectoral coordination platforms (PLATF4; 56.0\%) as low compared with international best practice.

Non-state actors rated the level of implementation of infrastructure support systems for food policy implementation higher than food policy indicators. The level of implementation compared with best practice was assessed as 'high' for two indicators, 'medium' for thirteen indicators, 'low' for fifteen indicators and 'very little, if any' for twelve indicators (Fig. 2). The latter ones are the indicators which were not rated by the experts as the level of implementation was considered nil by government officials. Among the food policy domains, the level of implementation of school and child-care centre policies on food environments (PROV1; 61.6\%) and front-of-pack labelling (LABEL3; 52.3\%) was rated the highest at 


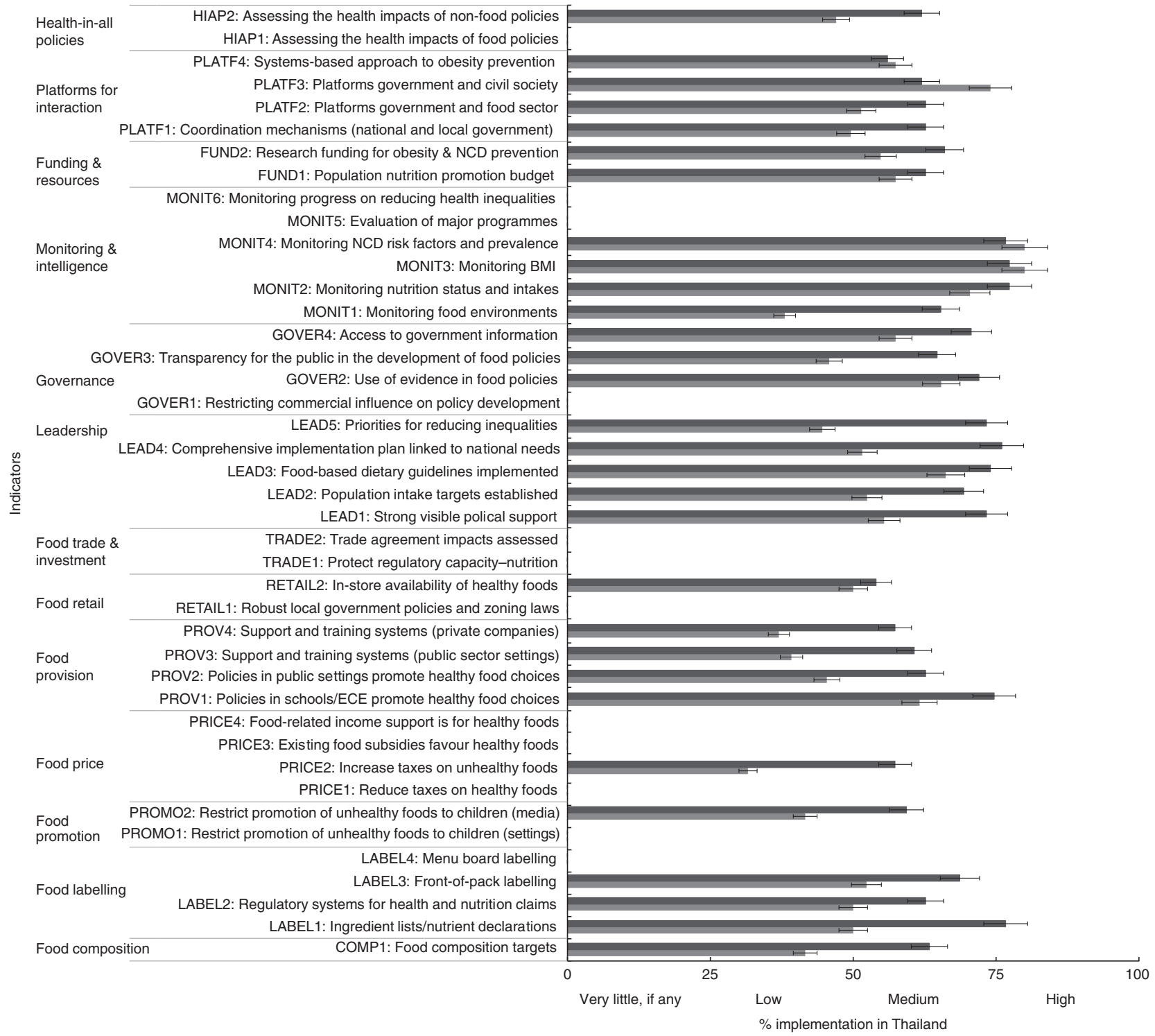

Fig. 2 Level of government implementation of food environment policies and infrastructure support for policy implementation by state $(\square)$ and non-state actors ( $\square$ ) in Thailand. Error bars show $5 \%$ positive and negative potential error amounts (NCD, noncommunicable disease; ECE, early childhood education)

'medium' implementation, while the level of implementation of taxes on unhealthy foods (PRICE2; 31.6\%) was rated as low. Among the infrastructure support indicators, monitoring NCD and risk factors and monitoring overweight and obesity (MONIT3 and MONIT4) were rated highest at $80 \%$ implementation while monitoring food environments (MONIT1; 38.0\%) was rated low.

Comparing the two groups of actors, there was a statistically significant difference in the rating scores of policy implementation between state and non-state actors (Wilcoxon rank-sum test, $P<0.05$ ). The state actors rated the implementation level across different individual domains with higher scores than non-state actors. Similarly, both state and non-state actors assessed better performance of the infrastructure domains than the food policy domains, except indicators on monitoring
NCD and risk factors (MONIT3), monitoring overweight and obesity (MONIT4), platforms for regular interactions between state and non-state groups (PLATF3) and implementing large-scale community-based approaches (PLATF4).

There were some discrepancies between both groups. The discrepancy that appeared to be largest was on priorities for reducing inequalities (LEAD5; 28.7\% difference), followed by monitoring food environments (MONIT1; $27 \cdot 3 \%$ difference), food labelling for ingredient lists/nutrient declarations (LABEL1; 26.7\% difference) and increasing taxes on unhealthy foods (PRICE2; $25.7 \%$ difference). The state actors rated the LEAD5 indicator with 'medium' implementation (73.3\%) while the nonstate actors rated it with 'low' implementation (44.6\%). Among these large discrepancies of assessment between 
the two groups, state actors rated a higher level of implementation than the non-state actors.

\section{Identifying and prioritizing concrete actions for the Thai Government}

Figures 3 and 4 show the four-quadrant scatter plots of prioritization of concrete actions proposed by state and non-state actors, respectively. Based on the implementation gaps from the rating results, the state actors recommended fifty-six actions across food policy ( $n$ 31) and infrastructure support ( $n$ 25) domains (see online supplementary material, Table S2) while the non-state actors recommended sixty actions across food policy ( $n$ 29) and infrastructure support ( $n$ 31) domains (Table S3).

Comparing the two groups, there were thirty similar proposed actions, with actions in the food labelling policy domain appearing to be most similar across the two groups. Among these thirty, only sixteen fell into the same

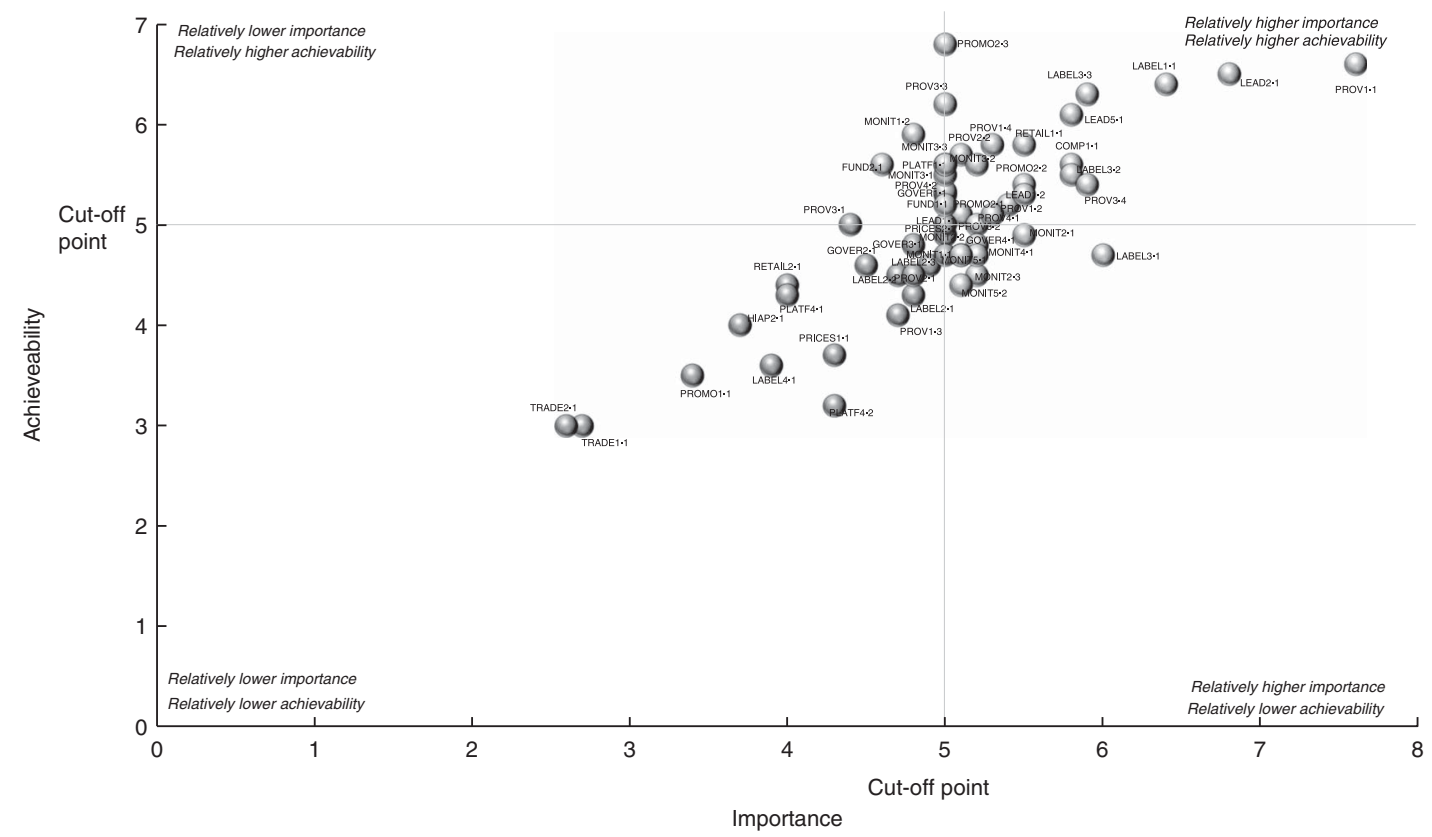

Fig. 3 Scatter plots of proposed actions, with relative importance of the action and relative achievability of the action, by state actors in Thailand

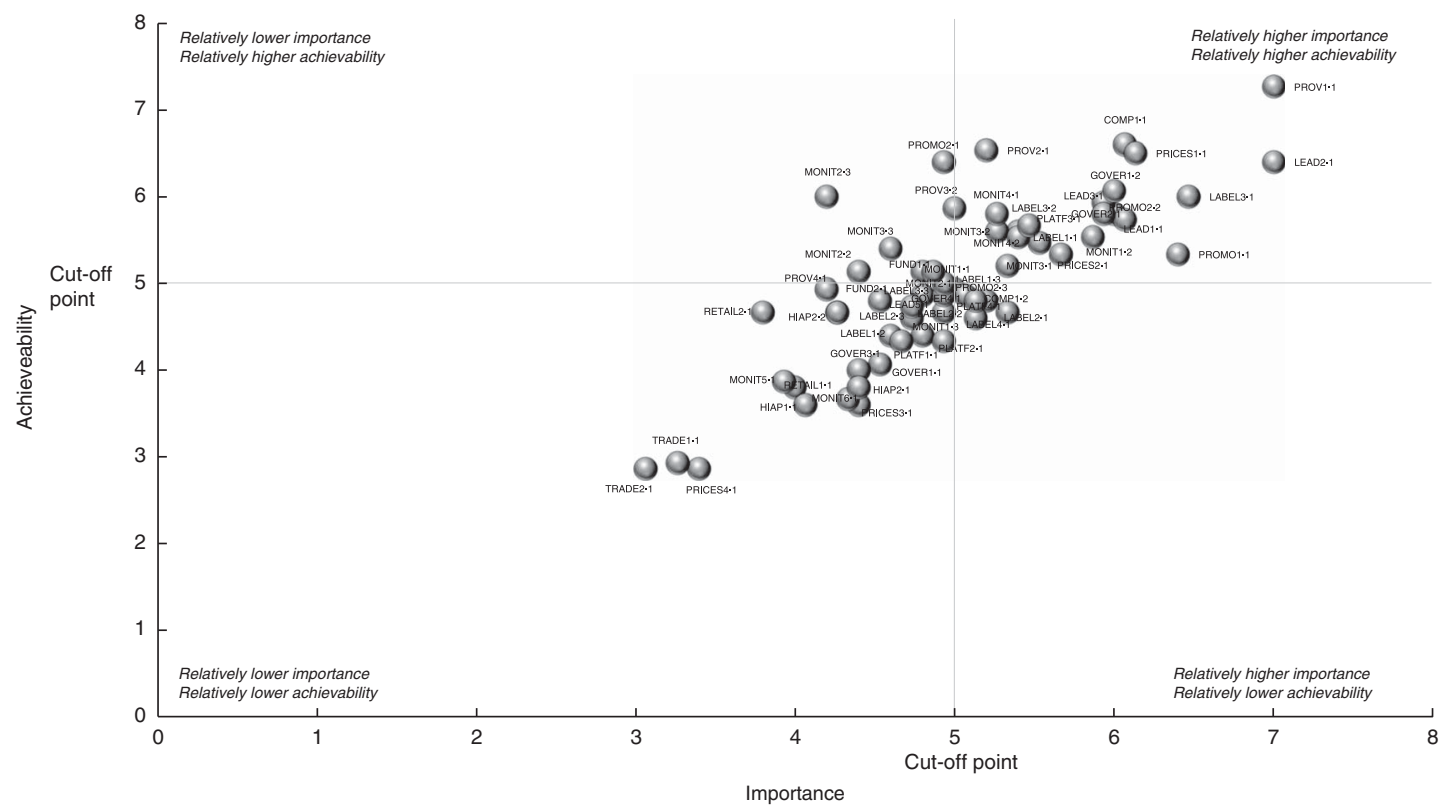

Fig. 4 Scatter plots of proposed actions, with relative importance of the action and relative achievability of the action, by non-state actors in Thailand 
Table 1 Consensus actions for the Thai Government reached by state and non-state actors in the joint meeting

\begin{tabular}{|c|c|}
\hline Domain & Consensus action(s) \\
\hline Food provision & $\begin{array}{l}\text { 1. The government should set standards for food and beverage products provided and sold in schools and child- } \\
\text { care centres, with efficient quality control and monitoring systems of food and beverage management complying } \\
\text { with the required standards. For example, the government can apply the standards of the 'Thai School Lunch' } \\
\text { programme focusing on the preparation of healthy lunch for young students to all child-care centres } \\
\text { 2. The government should promote provision and sale of healthy foods and drinks in hospitals and other public } \\
\text { settings }\end{array}$ \\
\hline Food labelling & $\begin{array}{l}\text { 3. The government should expand the display of ingredient lists and nutrient facts to more foods/food groups, } \\
\text { particularly those containing high sugar, saturated fat and salt, and request manufacturers to display these details } \\
\text { 4. The government should develop and improve a current nutrition information system to be understood easily and } \\
\text { clearly by the public and be evidence-informed, which allows consumers to make healthier food choices easily } \\
\text { 5. The government should develop a Thai nutrient profiling system and display of label according to a certain } \\
\text { nutrient profile } \\
\text { 6. The government should clearly determine required advertising standards for health and nutrition claims }\end{array}$ \\
\hline Food composition & $\begin{array}{l}\text { 7. The government should clearly indicate a required content of saturated fat, sugar and sodium added to major food } \\
\text { sources of these nutrients, that the content should be appropriate for people's daily lifestyle }\end{array}$ \\
\hline Food promotion & $\begin{array}{l}\text { 8. The government should clearly specify a marketing framework for foods and beverages, particularly promotional } \\
\text { strategies, and also review the development of advertising campaigns from international sources, especially the } \\
\text { promotional strategies used in schools and around school areas }\end{array}$ \\
\hline Leadership & $\begin{array}{l}\text { 9. The government should strongly commit to set its clearly defined goal in limiting intake of salt, sugar and } \\
\text { saturated fat among the Thai population by making use of } 6: 6: 1 \text { consumption criteria, meaning that a person is } \\
\text { suggested not to consume more than } 6 \text { teaspoons of saturated fat, } 6 \text { teaspoons of sugar and } 1 \text { teaspoon of salt } \\
\text { per day }\end{array}$ \\
\hline $\begin{array}{l}\text { Monitoring \& } \\
\text { intelligence }\end{array}$ & $\begin{array}{l}\text { 10. The government should request all educational institutions and other public settings to produce primary health } \\
\text { information, such as weight, waist circumference and blood pressure, of their related people and make follow-up } \\
\text { actions on update of such information. It is also required to keep people informed about their personal health } \\
\text { information and provide them efficient solutions for health problems }\end{array}$ \\
\hline $\begin{array}{r}\text { Food trade \& } \\
\text { investment }\end{array}$ & $\begin{array}{l}\text { 11. The government should clearly and carefully identify specific messages on the legal authorization of the Thai } \\
\text { Government according to terms and conditions of the international trade and investment agreement to prevent } \\
\text { NCD and promote the well-being of Thai people }\end{array}$ \\
\hline
\end{tabular}

NCD, non-communicable disease.

quadrants; eight similar actions were assessed with relatively higher importance and achievability (right-upper); one with having relatively lower importance, higher achievability (left-upper); and seven with relatively lower importance and achievability (left-lower). In the rightupper quadrant some indicator domains were proposed by one group, but not another. They were the actions on food price policy, governance and platform-for-interaction domains proposed by the non-state group, and the action on food retail policy domain proposed by the state group.

Forty-eight state and non-state actors attended a joint meeting that aimed to reach consensus on actions for the Thai Government. Eleven consensus actions were selected from the list of similar actions proposed from both groups. Of these eleven, eight were 'relatively higher important and achievable' actions, one was a 'relatively lower important, but relatively higher achievable' action, and two were 'relatively lower important and achievable' actions. Four actions focused on food labelling; two were on food provision; and one each was on food composition, food promotion, leadership, monitoring and intelligence, and food trade and investment (Table 1). The actions included setting standards for food and beverage products provided and sold in schools and child-care centres; promoting provision and sale of healthy foods and drinks in hospitals and other public settings; developing a Thai nutrient profiling system; establishing a marketing framework for foods and beverages, particularly promotional strategies; setting the government's clearly defined goal in limiting intake of salt, sugar and saturated fat among the Thai population; and developing self-monitoring systems in all educational institutions and other public settings to produce primary health information, such as weight, waist circumference and blood pressure, of their related people and make follow-up actions on update of such information.

\section{Discussion}

The present study found striking variations in the assessment of the level of implementation of government policies for creating healthy food environments by different stakeholder groups. Differences between the result of state actors and the result of non-state actors may be due to differences in perspectives of each other's aspects, especially their legitimate rights, role, capacity and motivation $^{(10-15)}$, which often impede the effective implementation of public policy. 
As a self-assessment, the state actors were more positive on the government's performance of policy implementation than non-state actors for the majority of indicators. These differences reflect different perspectives and values of the different stakeholders ${ }^{(10)}$. For example, it is possible that cognitive ${ }^{(16)}$ and/or motivational ${ }^{(13)}$ biases of state actors led to their more positive assessment. On the other hand, the non-state actors are, for the most part, not exposed to the 'on ground' implementation of the relevant policies, so they may not have had complete information regarding the level of implementation. Furthermore, nonstate sectors such as NGO have traditionally been at the forefront of advocating or catalysing governments to implement policies for change for civic interests ${ }^{(14)}$. Correspondingly, their high expectation for government action may have resulted in them making assessments at a lower level. Moreover, the level of understanding around the political economy and barriers to policy implementation was likely to be lower among non-state actors ${ }^{(11)}$. In the joint meeting of both groups, all actors were able to gain a better understanding of the limitations of government to take action. For example, the Department of Excise Tax was limited in its capacity to increase tax based on sugar levels in soft drinks as there is currently no clear policy coherence between trade, economic growth and health in Thailand.

To overcome differences in stakeholder's viewpoints on the implementation and avoid negative conflict from these differences, it is important for policy makers to understand the nature of individual stakeholders and groups of stakeholders that affect the policy implementation or are affected by the implementation at all levels. A stakeholder analysis can provide a means for understanding more about key actors' knowledge, interests, positions, alliances and importance related to the policy ${ }^{(17)}$. This analysis will allow the policy makers to interact more effectively with key stakeholders and increase support for the policy and its implementation. Importantly, the analysis should be carried out before implementing the policy so that the policy makers can detect and act to prevent potential misunderstandings and/or opposition to the policy implementation. To be more likely to make successful implementation, the stakeholder analysis should be used along with other key approaches especially the multisectoral and multi-stakeholder approach. First and foremost, stakeholders are to be realized as key to successful implementation, then a full range of potential stakeholders should be involved to help facilitate the implementation ${ }^{(6)}$ especially developing their understanding of and agreement with the policy and its implementation.

The adapted Food-EPI provided an opportunity and a mechanism to support a health-in-all policy approach which requires a health lens to be applied to all policies, as well as the development and implementation of effective cross-sectoral actions to improve population health and health equity ${ }^{(18)}$. The joint meeting offered a platform for interactive dialogue between stakeholders from health and non-health sectors, and between state and non-state actors, to enable sharing and understanding of institutional mandates and limitations, interests, power and positions, alliances and relationship with food industries. The meeting was successfully able to reach consensus on a single set of priority actions to be implemented by the Thai Government. This set of priorities will help to affirm joint commitments to take action and can be used to strengthen the accountability of all parties involved.

The prioritized list of actions from both groups includes normative functions of government in setting targets for fat, sugar and sodium contents in certain foods or food groups, setting food provision standards in schools, developing a nutrient profiling system and establishing a regulatory framework for food promotion. These normative functions are arguably less contentious and within the existing technical capacities of relevant agencies, without a need for cross-sectoral actions. They serve as a framework and reference point for the additional prioritized government interventions such as food labelling, food provision, food promotion and food taxation, which require more complex cross-sectoral actions.

As the Thai Government moves to implement these actions, it will remain essential for there to be institutional capacities in the regulatory bodies to enforce normative standards and ensure compliance by food industries, with effective sanctions for non-compliance.

The present study had strengths and limitations. The Food-EPI used was built on existing efforts at measuring political commitment and prioritization of concrete actions to overcome problems of unhealthy food environments that lead to obesity and diet-related NCD. Importantly, it provided an engagement process that brought government officials and non-government experts together in the process. However, the Food-EPI was designed specifically for the implementation stage of the policy cycle and therefore the explanatory power of the study was limited to this stage. Although the Food-EPI contributes to regular monitoring of progress that the government has made over time and will enable comparisons across countries, it does not aim to identify how and why the policies have or have not been successfully implemented as planned. Indeed, in order to increase the level of implementation, the Food-EPI may need to be complemented with other appropriate measures to assess the enabling factors and barriers for effective policy implementation, such as institutional capacities of responsible government agencies, regulatory capacities, governance, and transparency in preventing 'policy capture' whereby special interests exercise undue influence and capture the policy process $^{(19)}$.

Moreover, the present study examined only formal, national- and sub-national-level written policies as adopted by the relevant Ministries, the Thai Cabinet or by the central or provincial Superintendent's Office. Districts may 
have formulated additional policies through less formal approaches and these would not have been captured in the evidence summaries that documented current policy status. While it was explained to participants that the assessment of the level of implementation for each indicator required such assessments be based on the documented evidence, it is possible that participants factored other details into their assessments. More generally, it is recognized that participants could take into account multiple factors, including the quality of the policy as well as the extent of implementation, with reference to international best practice. Despite consistent instructions and guidance given to participants, and the strong reliability ratings of the tool, the complexity of the ratings tasks may have contributed to the differences in ratings observed.

The present study was implemented at a time of significant country reflection on strategies to address obesity and diet-related $\mathrm{NCD}^{(20)}$. It provided priority recommendations stemming from consensus between state and non-state actors. The process and set of agreed priorities will serve as a platform for helping to drive Thailand's future policy actions, facilitate greater collaboration between different stakeholders operating within and across sectors, and establish a baseline and targets to measure progress in the future. This forms a core part of a broader strategy for accelerating the government and stakeholders to be more responsive and accountable to their citizens ${ }^{(21)}$

\section{Acknowledgements}

Acknowledgements: The authors would like to thank the government officials and NGO representatives and academics in Thailand who participated in the rating workshops and the government officials who revised the accuracy and completeness of the evidence of policy implementation. The authors acknowledge the International Network for Food and Obesity/NCDs Research, Monitoring and Action Support (INFORMAS), the Food and Nutrition Policy for Health Promotion Program, International Health Policy Program Foundation, and Ms Nuttapat Makka and Ms Khanitta Kusreesakul from the Burden of Disease Thailand Program. Financial support: This paper was supported by the Thai Health Promotion Foundation (a not-for-profit statutory agency) and the WHO Thailand. S.P. received $\mathrm{PhD}$ research support from Deakin University Postgraduate Research Scholarship to complete this paper; otherwise has no financial disclosures. M.L., S.P. and G.S. are researchers within a National Health and Medical Research Council Centre for Research Excellence in Obesity Policy and Food Systems (APP1041020). G.S. is the recipient of an Australian Research Council Discovery Early Career Researcher Award (project number DE160100307). Conflict of interest: S.V., M.L., V.T. and G.S. have no financial disclosures.
The authors have no conflict of interest to report. Authorship: S.P. conceived the study and its original design, and drafted the initial form and all revisions of this paper. S.P. and V.T. collected the data. S.P., S.V., M.L., V.T. and G.S. reviewed and approved the final manuscript. Ethics of buman subject paticipation: This study was approved by the Human Ethics Advisory Group, Faculty of Health, Deakin University (HEAG-H 193_2014) and the Thailand Institution for the Development of Human Research Protection.

\section{Supplementary material}

To view supplementary material for this article, please visit http://dx.doi.org/10.1017/S1368980016002391

\section{References}

1. World Health Organization (2011) Global Status Report on Noncommunicable Diseases 2010. Geneva: WHO.

2. Davison KK \& Birch LL (2001) Childhood overweight: a contextual model and recommendations for future research. Obes Rev 2, 159-171.

3. Faith MS \& Kral T (2006) Social Environmental and Genetic Influences on Obesity and Obesity-Promoting Behaviors. Fostering Research Integration, Genes, Behavior, and the Social Environment: Moving Beyond the Nature/Nurture Debate. Washington, DC: National Academies Press.

4. Story M, Neumark-Sztainer D \& French S (2002) Individual and environmental influences on adolescent eating behaviors. J Am Diet Assoc 102, 3 Suppl., S40-S51.

5. World Health Organization (2014) Global Status Report on Noncommunicable Diseases 2014. Geneva: WHO.

6. Griffiths J, Maggs H \& George E (2008) Stakeholder Involvement. Background Paper Prepared for the WHO/WEF Joint Event on Preventing Noncommunicable Diseases in the Workplace. Geneva: WHO.

7. World Health Organization (2014) Global Status Report on Alcohol and Health - 2014. Geneva: WHO.

8. Swinburn B, Dominick CH \& Vandevijvere S (2014) Benchmarking Food Environments: Experts' Assessments of Policy Gaps and Priorities for the New Zealand Government. Auckland: University of Auckland.

9. World Cancer Research Fund (2013) WCRF international food policy framework for healthy diets: NOURISHING. http:// www.wcrf.org/policy_public_affairs/nourishing_framework/ \#about (accessed January 2014).

10. Carrin G, Buse K, Heggenhougen K et al. (2009) Health Systems Policy, Finance, and Organization. Oxford: Elsevier.

11. Damschroder L, Aron D, Keith R et al. (2009) Fostering implementation of health services research findings into practice: a consolidated framework for advancing implementation science. Implement Sci $\mathbf{4}, 50$.

12. Kunda Z (1990) The case for motivated reasoning. Psychol Bull 108, 480-498.

13. Molden DC \& Higgins ET (2012) Motivated thinking. In The Oxford Handbook of Thinking and Reasoning, pp. 390-409 [KJ Holyoak and RG Morrison, editors]. New York: Oxford University Press.

14. Piotrowicz M \& Cianciara D (2013) The role of nongovernmental organizations in the social and the health system. Przegl Epidemiol 67, 69-74, 151-155.

15. Durlak JA \& DuPre EP (2008) Implementation matters: a review of research on the influence of implementation on 
program outcomes and the factors affecting implementation. Am J Community Psychol 41, 327-350.

16. von Winterfeldt D \& Edwards W (1986) Decision Analysis and Behavioral Research. New York: Cambridge University Press.

17. Schmeer K (1999) Guidelines for Conducting a Stakeholder Analysis: Partnerships for Health Reform. Bethesda, MD: Abt Associates Inc.

18. Leppo K, Ollila E, Pena S et al. (2013) Health in All Policies. Seizing Opportunities, Implementing Policies. Helsinki: Ministry of Social Affairs and Health.
19. Joel SH, Geraint J, Daniel K et al. (2000) Measuring Governance, Corruption, and State Capture: How Firms and Bureaucrats Shape the Business Environment in Transit. Policy Research Working Paper no. 2312. Washington, DC: World Bank.

20. Bureau of Policy and Strategy (2015) Policy Agenda of Minister of Public Health Thailand in 2015. Nonthaburi: Ministry of Public Health Thailand.

21. Swinburn B, Kraak V, Rutter H et al. (2015) Strengthening of accountability systems to create healthy food environments and reduce global obesity. Lancet 385, 2534-2545. 